P. Schadewaldt

\title{
Investigation of branched-chain amino acid metabolism in man using stable isotopes
}

\section{Stoffwechsel verzweigtkettiger Aminosäuren}

The branched-chain amino acids (BCAA), L-leucine, Lvaline, and $\mathrm{L}$-isoleucine, comprise about $40 \%$ of the essential amino acids. The main metabolic fate is incorporation into body protein. Depending on the (patho)physiological state, variable amounts may be degraded and serve fuel functions.

In recent years, availability of stable-isotope techniques has prompted an increasing number of investigations on the quantitative and regulatory aspects of BCAA metabolism in man. The primed continuous infusion of $\mathrm{L}-\left[1-{ }^{13} \mathrm{C}\right]$ leucine has become the method of choice. Reports on studies applying compartmental approaches, i.e., venous or oral bolus tests, or other BCAA substrates are rare. Thus, data on human BCAA metabolism are largely restricted on leucine metabolism as evaluated under experimental steady state conditions.

In summarizing accumulated data, the most important physiological variables eliciting long- term and shortterm effects on leucine turnover in vivo are provision of
P. Schadewaldt

Diabetes Forschungsinstitut an der

Heinrich-Heine-Universität Düsseldorf

Auf'm Hennekamp 65

D-40225 Düsseldorf

Germany substrate by the diet and fasting and physical exercise, respectively. In a concentration dependent manner, an increase in substrate intake is primarily diverted into protein the relative excess being removed by an increase in whole body leucine oxidation rate (WBLO). Deprivation of exogenous leucine is at least partially compensated by a decrease in WBLO. In accordance, leucine turnover in healthy volunteers has been shown to exhibit significant diurnal changes under normal conditions.

Overall leucine balance, however, not necessarily reflects net nitrogen balance. This is presumably due to functionally different protein pools and to specific changes in the relation of the anabolic and catabolic rates of leucine turnover, which do not parallel changes in whole body protein metabolism.

The most marked short-term effects are noted during aerobic muscular exercise which causes a several fold enhancement in WBLO. Obviously, this is referable to an increase in the activity state of the branched-chain 2-oxo acid dehydrogenase complex in working muscle tissue. Several studies failed, however, to detect effects of resistance training programs.

With respect to hormonal regulation, specific effects are, for instance, indicated by the finding that $\beta$-blockade leads to a remarkable increase in WBLO during muscular exercise. Most hormones investigated, e.g., insulin, GH, IGF- 1, androgens, and glucocorticoids, are now thought to influence BCAA turnover primarily by indirect means, however, via their effects on protein and energy metabolism.

Taken together, a considerable extension of our knowledge on leucine kinetics has accumulated from stable isotope studies. However, further studies may still be necessary towards a better understanding of human BCAA metabolism in vivo. For instance, the quantitative significance of individual organs, the interorgan relationships, and possible differences in the handling of the individual BCAA are not well defined at present and remain to be investigated. 